\title{
Les espaces scolaires en France
}

Table ronde

School as spaces in France. Round table

Los espacios escolares en Francia. Mesa redonda

Maurice Mazalto, Luca Paltrinieri, Bernard Quirot, Florence Robine, Philippe Tournier et Henriette Zoughebi

\section{OpenEdition \\ Journals}

Édition électronique

URL : https://journals.openedition.org/ries/3615

DOI : 10.4000/ries.3615

ISSN : 2261-4265

Éditeur

France Education international

Édition imprimée

Date de publication : 1 décembre 2013

Pagination : 77-91

ISBN : 978-2-85420-601-2

ISSN : $1254-4590$

Référence électronique

Maurice Mazalto, Luca Paltrinieri, Bernard Quirot, Florence Robine, Philippe Tournier et Henriette Zoughebi, «Les espaces scolaires en France », Revue internationale d'éducation de Sèvres [En ligne], 64 I décembre 2013, mis en ligne le 01 décembre 2015, consulté le 28 juin 2022. URL : http:// journals.openedition.org/ries/3615; DOI : https://doi.org/10.4000/ries.3615 


\title{
Les espaces scolaires en France
}

\section{Table ronde}

\author{
Maurice Mazalto \\ Luca Paltrinieri \\ Bernard Quirot \\ Florence Robine \\ Philippe Tournier \\ Henriette Zoughebi
}

Cette table ronde s'est tenue au Centre international d'études pédagogiques le 25 septembre 2013. Elle a réuni: Henriette Zoughebi, vice-présidente du Conseil régional d'Île-de-France, en charge des lycées et des politiques éducatives (470 lycées publics, 205 lycées privés, pour une population de 800000 lycéens du public et 76000 apprentis) ; Bernard Quirot, architecte, qui a enseigné à l'École d'architecture de Paris/Belleville et qui réalise des groupes scolaires et des collèges; Florence Robine, rectrice de l'académie de Créteil, qui a eu les mêmes responsabilités à Rouen et en Guyane; Philippe Tournier, proviseur du lycée Victor Duruy à Paris, secrétaire national du Syndicat national des personnels de direction de l'Éducation nationale. L'animation de la table ronde était assurée par les coordinateurs du numéro 64 de la Revue internationale d'éducation de Sèvres, consacré aux espaces scolaires : Luca Paltrineri, philosophe et Maurice Mazalto, ancien proviseur. NDLR

Maurice Mazalto : Il serait vain de vouloir tracer, en quelques minutes, l'historique de l'école en France et des espaces qui la composent. On peut simplement souligner que l'école suit l'évolution de la société française : elle en est le reflet, elle en adopte les principes. Ainsi, pendant très longtemps, l'enseignement a été l'apanage de l'Église, avec la construction de collèges, dont certains existent encore aujourd'hui.

Le Premier empire a créé des écoles secondaires et des lycées sur des modèles architecturaux souvent militaires. La Troisième République, souhaitant l'émancipation de tous par l'instruction, a réalisé un effort colossal pour doter chaque village d'une école. En vingt ans, 15000 écoles ont été construites, 30000 ont été rénovées. Cette Troisième République a construit également construit des bâtiments plus prestigieux dans les villes, de nouveaux temples dédiés à la science.

Après la Seconde Guerre mondiale, des évolutions démographiques et l'instauration du collège unique incitent les responsables de l'époque à développer des constructions industrielles normalisées au moindre coût: 3500 collèges sont construits en dix ans, à partir de 1966. 
Le grand bouleversement intervient dans les années quatre-vingts, avec la promulgation des lois de décentralisation qui transfèrent les responsabilités aux collectivités territoriales, développant ainsi une architecture plus adaptée à son territoire, mieux insérée dans son environnement. Il en résulte une grande diversité des espaces scolaires.

Aujourd'hui, les projets éducatifs se multiplient pour favoriser la réussite du plus grand nombre, grâce, notamment, à des pédagogues novateurs; des études soulignent l'importance des espaces scolaires sur l'efficacité des acquis, le climat général dans l'établissement, la qualité des comportements, l'ouverture sur l'extérieur.

Après avoir précisé la notion d'espaces scolaires, il conviendra de s’interroger sur les relations qui existent entre ces espaces et les projets éducatifs, sur les réponses qui peuvent être envisagées pour répondre aux défis actuels, et d'aborder l'avenir avec l'introduction des nouvelles technologies. Comme l'affirmait l'architecte visionnaire Claude Nicolas Ledoux au XVIII ${ }^{\mathrm{e}}$ siècle, « la qualité du cadre de vie conditionne la pensée et le comportement ».

\section{QUELLE DÉFINITION DES ESPACES SCOLAIRES?}

Luca Paltrinieri: Pour commencer, quelle définition des espaces scolaires êtes-vous amenés à donner dans votre travail, et dans quelle mesure cette question recoupe-t-elle une préoccupation pédagogique et un projet éducatif précis? Dans la brève présentation par Maurice Mazalto de l'histoire de l'espace éducatif en France, on peut imaginer que chaque évolution de l'espace éducatif sous-tend un projet éducatif donné, ou une certaine conception de la pédagogie. Quelle est votre définition de l'espace, du bâtiment, de la salle de classe et comment, d'une façon ou d'une autre, résume-t-elle le projet éducatif que vous avez chacun, ainsi que les projets auxquels vous prenez part, selon vos responsabilités respectives au sein de la communauté éducative?

Philippe Tournier: L'espace, ce sont des mètres carrés. D’expérience, je peux dire que les ratios du taux d'occupation sont une contrainte qui perturbe très fortement les activités dans les établissements. Lorsqu'un établissement est constamment occupé, les élèves et les professeurs changent sans arrêt de salle, personne n'a un espace qui lui est propre et le mode de fonctionnement reste fortement indifférencié.

Ce qui est stable, dans nos espaces scolaires, c'est le couloir, avec des salles de classe de $65 \mathrm{~m}^{2}$ où il y a 35 places, les unes derrière les autres. Ce qui est nouveau, innovant, ne concerne que les espaces périphériques : soit les halls d'accueil (fortement mis en valeur au XIX ${ }^{\mathrm{e}}$ siècle, oubliés pendant l'âge fonctionnel, à nouveau remis en valeur depuis la décentralisation) ou des espaces comme des cafétérias, des salles de travail pour les professeurs. Je constate aussi 
que, lorsque la pression démographique se fait sentir dans un établissement, ces espaces sont systématiquement sacrifiés au profit du noyau central, celui de la salle de classe. Ce dernier, avec ses 35 places alignées en rangs d'oignons, est l'expression physique exacte du fonctionnement réel du système éducatif.

L'expérience montre que configurer différemment des espaces ne suffit pas à modifier des pratiques. J'en prends deux exemples. Dans les années soixante-dix, l'État, sans doute bourrelé de remords après les conceptions fonctionnelles précédentes, a construit près de Dunkerque, à la grande époque d'Usinor, un lycée qui se voulait un lycée de la transparence : toutes les salles de classe étaient vitrées, afin que tout le monde puisse vivre ensemble. Au même moment, on a construit à Villeneuve d'Ascq, dans la banlieue de Lille, le lycée Raymond Queneau, avec l'idée que les citoyens pourraient en traverser le centre, que les élèves et les habitants s'y rencontreraient. Aujourd'hui, à Dunkerque, les salles sont occultées parce qu'il était insupportable pour les professeurs et les élèves d'être constamment sous le regard d'autrui ; quant à Villeneuve d'Ascq, la place a été grillagée et aujourd'hui, il faut faire le tour du lycée. Cela veut dire que l'architecture ne modifie pas les pratiques. Les gens s'approprient les espaces tels qu'ils fonctionnent et l'on peut constater qu'aujourd'hui, le seul espace réellement légitime dans l'imaginaire et dans le fonctionnement concret du système éducatif, c'est la traditionnelle salle de classe.

Luca Paltrinieri : Entre l'architecture et les pratiques, il existe donc un troisième élément qui pourrait être l'espace en tant que production sociale des différents acteurs qui se croisent à l'intérieur ou à l'extérieur de l'école en tant que bâtiment?

Philippe Tournier: On construit toujours pour une période qui excède le temps de nos croyances pédagogiques. C'est d'ailleurs pour cela que, finalement, une sorte de norme implicite finit par s'imposer. Actuellement, par exemple, on intègre la question des espaces numériques de travail et des évolutions technologiques contemporaines. Mais que sait-on d'eux dans un siècle? On n'en n'a aucune idée.

Florence Robine : Ce qui structure, à l'heure actuelle, les établissements scolaires et montre bien la façon dont notre système lui-même est structuré, ce sont les espaces d'enseignement fondamentaux: l'enseignement traditionnel, frontal, en salle de classe, avec le maître devant les élèves. C'est considéré comme le cœur des enseignements et donc de l'espace, le reste n'étant que périphérique et dépendant de conceptions diverses et variées, momentanées, de ce qui fait le "supplément d'âme» de l'éducation. C'est peut-être cela qu'il faut changer. D'autre part, ce qui caractérise également ces espaces, c'est leur aspect fermé et cloisonné. Nombre de petits espaces, de salles de classe, sont fermés sur 
eux-mêmes. Enfin, je voudrais souligner qu'il faut penser un projet plus global, faute de quoi tous ces suppléments d'âme, toute cette organisation périphérique deviendront très vite obsolètes par rapport aux doctrines pédagogiques ou didactiques en vigueur. Il faut parvenir à dé-corréler un certain nombre de préidentifications ou de prédestinations faites par l'institution, et par elle seulement. Par exemple, à une époque où il était hors de question de mélanger les disciplines, il y avait le laboratoire de biologie et celui de physique, si possible chacun à un bout du bâtiment, si bien que les enseignants ne se rencontraient pas. Finalement, ces structures prédisposent la façon de travailler. Comment faire en sorte que les usagers ne soient pas contraints de trouver leur place dans des organisations préexistantes mais puissent être véritablement partie prenante de la destination, de l'organisation des lieux en fonction des projets pédagogiques?

Henriette Zoughebi : Un lycée ancien situé à Paris, où le foncier est très cher, ne pose pas les mêmes problèmes qu'un établissement situé en Seine-etMarne, dans un espace plus rural. Les contraintes existent toujours et font partie de la règle du jeu. Ce qui me frappe, dans l'évolution décrite par Maurice Mazalto, c'est que nous ne sommes plus au temps où l'on avait pour modèle prégnant un modèle d'État. Outre les évolutions liées à la décentralisation, on observe aujourd'hui de nouvelles aspirations de la société, et c'est intéressant d'y réfléchir. L'introduction de la notion de programmes de construction me paraît à cet égard extrêmement importante, car elle tient compte à la fois de la pédagogie, des possibilités qu'offre le terrain et de la concertation avec les usagers, ce qui est nouveau. Le Conseil régional d'île-de-France a voté, en juin 2011, l'obligation d'un système de concertation avec les communautés éducatives, assez contraignant pour les services mais extrêmement important parce qu'il permet d'être au plus près des attentes.

Je voudrais également souligner que nos sociétés vivent un moment de bascule. Par exemple, quelle place les lycéens vont-ils avoir dans les lycées ? Pour moi, c'est une question fondamentale. Pour des raisons qui tiennent à l'évolution même des savoirs et de la transmission, on ne peut pas conserver l'aspect descendant qui existe encore aujourd'hui. Dans sa salle de classe, le professeur n'est pas le seul à détenir un certain nombre d'éléments de pouvoir. Les élèves peuvent trouver de l'information ailleurs, en consultant Wikipédia sur Internet, etc. Par ailleurs, notre société a de nouvelles exigences, par exemple celle du développement durable comme éthique. Comment l'espace raconte-t-il une histoire qui prépare les jeunes à prendre en compte toutes ces données?

Enfin, je voudrais insister sur le rapport entre l'espace du lycée et l'espace public extérieur, celui de la ville. Je pense que cette notion est également en train d'évoluer. J'aimais beaucoup l'esthétique et la structure des vieux lycées aux grandes fenêtres carrées. Aujourd'hui existe une volonté que les lycées 
deviennent vraiment des " gestes " architecturaux dans la cité, et je suis sensible à cet aspect. C'est un espace qui doit donner du sens à l'ensemble de la collectivité qui vit autour, au-delà du quartier. L'idée que la beauté donne de la dignité aux jeunes qui fréquentent l'établissement me paraît très importante. Tous les jeunes que je rencontre me disent combien ils se sentent eux-mêmes valorisés par cela. Quand un lycée professionnel est rénové, qu'il a du beau matériel, de l'allure, ceux qui y viennent le regardent différemment et ne sont pas regardés de la même manière. Les humains qui vivent dans ces espaces doivent pouvoir se prononcer sur ce qui s'y passe et doivent y trouver leur part de valorisation, à travers l'architecture.

Luca Paltrinieri: Bernard Quirot, vous qui êtes architecte, observezvous un rapport entre un beau bâtiment et la réussite des élèves ? S'agit-il d'une relation directe?

Bernard Quirot : Effectivement, l'architecture est d'abord là pour offrir de la dignité à ceux qui vont utiliser les bâtiments. Une bonne architecture dépend beaucoup de la qualité de la construction, ce qui n'a pas été le cas des établissements scolaires pendant très longtemps. Pourtant, l'architecture, c'est d'abord l'art de construire et, dans ce sens, elle peut être un modèle pour les élèves qui y demeurent. Comme l'a remarqué $M$. Tournier, il est en effet très surprenant que depuis le XIX ${ }^{\mathrm{e}}$ siècle, le noyau dur des établissements, la salle de classe, n’ait pas évolué. Je dirais même que cet espace a plutôt été dévalué au fil $\mathrm{du}$ temps. Pourquoi les nouvelles technologies n'ont-elles pratiquement aucune incidence sur la définition de cet espace, pourquoi les programmes de construction restent-ils peu ou prou les mêmes et pourquoi, cela a été souligné fort justement, les seuls espaces à faire l'objet d'une recherche, d'une petite évolution, sont-ils ces espaces de vie scolaire, comme on les appelle généralement? Il ne m'appartient pas d'y répondre.

En ce qui concerne les lycées anciens, il faut rappeler l'importance du couple salle de classe/espace de distribution. Ce dernier a longtemps été conçu comme un espace vaste, bien éclairé, qui apportait un surplus de lumière aux salles de classe. Il y a eu toute une recherche dans la lignée de l'hygiénisme, qui s'est traduite par une grande qualité de l'espace de la salle de classe. Petit à petit, dans les programmes, cette qualité s'est appauvrie, notamment en ce qui concerne la hauteur sous plafond, la lumière naturelle, etc. Aujourd'hui, le ratio de surface de circulation dans un lycée est d'environ $20 \%$; le plus souvent, on emploie du PVC pour le sol et des éléments modulaires pour les plafonds. La hauteur sous plafond est de trois mètres au maximum. Dans ces conditions, la générosité et la dignité sont bien difficiles à retrouver et si l'architecture se montre parfois incapable d'accueillir de nouvelles pratiques, c'est qu'elle est devenue trop pauvre pour cela. 
Le développement durable est une chance, en quelque sorte, non pas sous ses aspects thermiques qui sont parfois exagérés, mais pour redonner une qualité à cet espace premier qu'est la salle de classe, notamment à travers les réflexions sur la qualité de l'air, de la lumière naturelle. Grâce à ces arguments, il est possible aujourd'hui, par exemple, de convaincre un maître d'ouvrage de faire des salles de classe de 4 ou 5 mètres sous plafond, comme autrefois.

Par ailleurs, si le lycée, l'école, le collège doivent en effet s'inscrire comme monuments dans la ville, ou en tout cas comme institutions, c'est le logement qui en est le noyau dur comme la salle de classe est celui des espaces scolaires. Beaucoup de gestes architecturaux ont été produits ces dernières années. Sont-ils toujours d'une bonne qualité architecturale et sont-ils bien construits? Je n'en suis pas certain, mais il faut remarquer toutefois que ces « efforts » architecturaux portent souvent sur les espaces de la vie scolaire et non sur la salle de classe.

\section{DES ESPACES DE SOCIALISATION}

Maurice Mazalto: La question qui se pose aujourd'hui est-elle: comment passer d'établissements "machines à enseigner » à des établissements qui favorisent la socialisation, le "vivre ensemble »? Quelles sont les résistances à ce type d'évolution?

Bernard Quirot : La concertation a ses limites, mais reste indispensable. Concevoir et construire un bâtiment sans échanger avec ses futurs utilisateurs est évidemment impossible. Un bâtiment livré sans concertation est un bâtiment généralement mal accepté. Cette concertation, en tout cas dans ma pratique professionnelle, n'existe pratiquement pas. Les utilisateurs sont souvent perçus comme des gêneurs, et c'est évidemment très dommageable pour la qualité des bâtiments.

Florence Robine : Je crois que se dessine de plus en plus, même si cela ne fait pas encore consensus, une conception de l'espace scolaire comme véritable objet d'éducation, lieu de socialisation, levier pour former à la citoyenneté. La citoyenneté est désormais au cœur du projet de la société française, de l'idée républicaine de l'école. Nous devons, une fois que nous disposons des bâtiments, quels qu'ils soient, associer l'ensemble de la communauté éducative à l'utilisation de l'espace, à la façon dont on peut repenser toute l'organisation pédagogique globale de l'établissement en fonction de ces utilisations possibles de l'espace par chacun.

J'ai souvent été frappée, aussi bien dans ma fonction actuelle qu'en tant que parent, que les élèves soient parfois considérés comme des éléments perturbateurs de l'école : on a besoin d'eux, ils sont là mais il faut qu'ils restent figés dans une salle de classe, à un moment donné, à un instant « $T$ ». Ce qu'ils font 
en dehors du temps sacralisé de l'heure de cours, pendant leurs déplacements, ces temps considérés comme "morts", "perdus» sont justement les temps pendant lesquels le face à face pédagogique frontal avec l'enseignant n'existe plus. J'aspire - et je ne suis pas la seule - à ce que ces moments deviennent des temps fructueux, utiles, des temps qui participent à ces idéaux d'éducation, de socialisation, de responsabilisation des jeunes. Finalement, en tant qu'élève, parent, enseignant, membre de l'administration, comment chacun peut-il prendre sa part à la gouvernance de l'établissement, à ses choix, à la façon dont on organise le travail, à la façon dont on interagit et avec qui ? Il y a tout un travail à faire à partir de cette nouvelle conception de l'espace scolaire.

Philippe Tournier: La société est dans un moment de bascule mais l'école n'a pas encore basculé. Ce qui la caractérise aujourd'hui, c'est un comportement apeuré vis-à-vis du monde qui l'entoure. On fait la chasse actuellement, dans les établissements scolaires français, aux portables, à Wikipédia, car ils seraient illégitimes. On assiste aujourd'hui à des formes de repli identitaire dans l'école - ce que je dis n'est pas très optimiste - attisées par l'émergence de problématiques qui ont pesé lourd sur les choix architecturaux, comme les questions de sécurité. Dans les années soixante-dix, ces questions ne se posaient pas ou n'étaient pas appréhendées de la même manière. Aujourd'hui Il est plus difficile d'entrer dans un lycée parisien qu'il y a quarante ou cinquante ans. Certaines évolutions poussent, au contraire, à l'exaltation du caractère propre de l'école, qui est la salle de classe. C'est d'ailleurs pourquoi la concertation nécessaire peut s'avérer décevante, lorsque les personnes consultées évoquent un passé ou un présent anobli, sans réellement se projeter dans l'avenir. Tout récemment encore, il était difficile d'expliquer que l'ensemble des bureaux de l'administration générale devait être regroupé autour d'un seul point d'accueil et non pas séparé entre «l'administration » d'un côté et «l'intendance » de l'autre.

Ce qui me frappe également, si l'on compare la situation actuelle avec le passé, c'est de constater qu'un emploi du temps sous Louis-Philippe, par exemple, comportait des temps de cours bien moins nombreux qu'aujourd'hui, et des temps de permanence, de répétition, etc., qui étaient conçus comme des temps personnels de l'élève. Aujourd'hui, l'école n'est plus que du cours devant un professeur, les autres temps étant plus ou moins légitimes, sans compter qu'on peut se demander à quoi sert une cour de récréation dans un lycée aujourd'hui, puisque la principale activité des élèves, pendant ce moment de liberté, est, semble-t-il, de téléphoner.

La concertation peut donner des résultats très décevants dans ce contexte mais, d'un autre côté, fabriquer des espaces qui ne correspondent pas à ce que les gens attendent ne transformera pas leurs pratiques. D'une certaine manière, je plaiderais plutôt pour des espaces neutres et flexibles, qui ne fassent pas opposition aux évolutions qui finiront bien par avoir lieu. 
Henriette Zoughebi : Tout dépend de ce qu'on appelle concertation. Il faut commencer ce processus très en amont, en mettant en place des procédures d'information et de concertation, dans le respect de l'institution scolaire et de l'ensemble de la communauté éducative, pour se mettre d'accord sur les modalités de travail et sur des objectifs clairs. Ce qui est fondamental, ce sont les objectifs du système scolaire: qu'attend aujourd'hui de son école la société française, et à partir de cela, comment construire des espaces qui permettront d'atteindre ces objectifs? Si l'on pense - et j'en suis convaincue - que tous les enfants peuvent réussir, l'école doit changer son mode de fonctionnement par rapport à cet objectif. Je crois que le CDI, qui va devenir le centre de connaissance et de culture $(3 \mathrm{C})$, jouera dans l'avenir un rôle central à l'intérieur d'un établissement. Si l'on pense les jeunes comme des citoyens et des citoyennes, les espaces de vie ne sont pas les mêmes. Si l'on pense que la culture n'est pas un accessoire de l'éducation mais est centrale parce qu'elle apporte la confiance en soi, donne de l'énergie aux jeunes, de la curiosité de découvrir, d'apprendre, la salle polyvalente n'est plus périphérique.

C'est pourquoi le rôle des programmistes est important : il faut travailler en tenant compte des contraintes du système - je suis favorable à l'idée que des cadres soient donnés au niveau national -, mais favoriser ensuite l'ouverture et la concertation. Les élus doivent rester extrêmement modestes. Ils assument une grande responsabilité en apportant les moyens et ils doivent également percevoir les changements dont les jeunes ont besoin. Il y a de l'inconnu dans tout cela. C'est pourquoi cette notion de modularité me semble aussi importante. Aujourd'hui, l'école n'a pas les certitudes qu'elle avait sous Jules Ferry. Intégrer cette part d'inconnu me paraît indispensable et extrêmement stimulant, à condition que l'objectif de la réussite pour tous soit celui vers lequel s'orienteront tous les efforts : ceux de l'éducation nationale dans toute sa diversité, ceux des collectivités et des hommes et des femmes de l'art.

Bernard Quirot: Ces évolutions sont quand même très minces. Aujourd'hui, ce qu'on demande à un architecte, c'est éventuellement la modularité de la salle de classe, par exemple qu'elle ne soit pas entravée par des murs porteurs afin de pouvoir faire évoluer légèrement sa surface. Il est toujours difficile de faire évoluer un bâtiment. En réalité, peu de demandes traduisent cette nouvelle vision de l'école. Je ne suis pas certain que la décentralisation, paradoxalement, ait eu un impact positif, dans la mesure où il manque une force centralisatrice capable d'impulser de nouveaux bâtiments. L'État ne mène plus une politique de qualité architecturale et on observe ainsi, sauf dans quelques régions, où les élus remplissent bien leur rôle, très peu d'innovations, très peu de désir de construire une école nouvelle.

Florence Robine: Il s'agit de définir la bonne place de l'État. Je suis convaincue, au contraire, que le travail avec la région Île-de-France nous a 
permis, à Créteil, de définir des structures, des projets plus proches de réalités extrêmement diversifiées, et qui ne sont pas nécessairement perçues comme telles par le niveau central. Les problématiques des lycées de Paris, par exemple, ont très peu à voir avec celles des lycées de la Seine Saint Denis ou de la Seine et Marne. Je pense que le rôle de l'État est d'exposer clairement les objectifs de l'école, en menant un travail de proximité avec les usagers pour faire partager ces objectifs et permettre qu'ils se les approprient.

En revanche, je ne partage pas l'idée qu'on ne voit pas d'évolution et que, globalement, les établissements n'ont que peu changé. Je pense à un établissement scolaire de la Seine-Saint-Denis ou à un autre en Seine-et-Marne, dans une région assez lointaine, où la difficulté scolaire constatée par les enseignants et par l'équipe éducative, la nécessité de faire réussir tous les élèves, de faire de la différenciation pédagogique, de s'intéresser aussi aux difficultés sociales des familles a tout naturellement conduit les équipes pédagogiques à s’interroger sur les formes scolaires, sur la façon dont on accompagne ces réussites, et à repenser les temps et les emplois des temps des élèves, sous toutes leurs formes. Cela a conduit à une autre organisation des apprentissages et de l'occupation de l'espace.

Nous avons désormais besoin d'espaces flexibles, modulaires, de concevoir autrement les centres de documentation, qui deviennent des centres de connaissances et de culture et occupent une position beaucoup plus centrale. Quelle part donner au numérique, aux outils, à la mobilité, aux espaces de détente ou de rencontre des élèves, quel temps en dehors du temps frontal d'enseignement ? Il faut réfléchir à de nouvelles organisations des apprentissages, du temps scolaire mais aussi des espaces scolaires, qui permettent cette adaptation aux problématiques spécifiques qui sont identifiées par les usagers de l'école.

La concertation doit exister en amont de la construction, bien sûr, mais aussi en aval, à travers le projet pédagogique d'établissement où l'on interroge tout le monde, et les élèves en particulier, sur ce qu'ils font dans les établissements. Que font les élèves toute la journée à l'école? Quand on veut bien prendre la peine de les interroger et de les écouter, on s'aperçoit qu'ils passent $80 \%$ de leur temps dans l'école et $20 \%$ à dormir ou dans les transports : c'est cela la réalité.

Maurice Mazalto : J'ai mené une enquête en 2012 auprès de 830 élèves du second degré, de la $6^{\mathrm{e}}$ à la terminale, provenant de cinq collèges et lycées répartis sur la métropole, comprenant $46 \%$ de filles, et portant sur leurs activités durant les moments de pause et de détente, en dehors des heures de cours. En ce qui concerne le corps en mouvement au collège, courir concernait $40 \%$ des élèves de $6^{\mathrm{e}}$ (davantage les filles) mais seulement $18 \%$ des autres niveaux et était inexistant au lycée. En ce qui concerne le corps au repos, au collège, $40 \%$ déclaraient se reposer, davantage les filles, avec un pic à $60 \%$ en $3^{\mathrm{e}}$, confirmé au 
lycée à $65 \%$. En ce qui concerne les relations sociales, 80 à $90 \%$ déclaraient échanger avec un/une ou plusieurs camarades, de la $6^{\mathrm{e}}$ à la terminale, sans différence notable entre le collège et le lycée. Le fait de travailler seul ou à plusieurs concernait 10 à $15 \%$ des élèves (35\% pour les filles au lycée).

Si l'on tient compte de ces données, et sachant qu'un élève de $6^{\mathrm{e}}$ et un élève de $3^{\mathrm{e}}$ n'ont pas les mêmes demandes, besoins, envies, ne faudrait-il pas réfléchir à l'organisation des espaces scolaires, en introduisant par exemple la notion de territoire? Ne faudrait-il pas envisager des aménagements favorisant les processus de socialisation, développer des espaces qui permettent les rencontres, les échanges, comme par exemple, des gradins, des odéons, des tables et des bancs d'extérieurs?

Henriette Zoughebi : Pour avoir fait depuis 2011 déjà deux concertations sur les attentes des jeunes et en avoir lancé une nouvelle en direction de 400000 lycéens de lycées publics, en collaboration avec les trois rectorats concernés, je peux dire que tous les jeunes déclarent que le lycée est leur deuxième maison, et cela rejoint ce que vous avez constaté.

Deux points m'intéressent particulièrement dans votre enquête. Le premier, c'est le rapport au corps, souvent mal pris en considération en France. Le fait que des élus de proximité soient interpellés sur l'état du collège, du lycée, a accéléré de manière considérable les constructions et les rénovations. Il faut savoir que la Région Île-de-France possède un patrimoine de 6,5 millions de mètres carrés - c'est l'un des constructeurs publics les plus importants d'Europe - et a engagé des opérations lourdes sur les deux tiers de ses lycées. L'État n'aurait pas pu le faire. Je veux dire par là qu'il y a eu une accélération, parce qu'il y a eu un rapprochement entre les citoyens et les élus. Concernant les salles de sports, les gymnases, pour lesquels il n'y a pas d'obligation légale - il existe des lycées sans terrain de sport -, nous venons d'intégrer cette dimension dans nos programmes. En Île-de-France, il est désormais obligatoire d'avoir au moins une salle de sports ou un gymnase. Nous avons fait évoluer nos référentiels pour tenir compte des attentes des jeunes, pour considérer davantage le rapport au corps, dès l'école.

Le deuxième point porte sur les lieux de socialisation. On a fait voter en 2012 un budget participatif pour les lieux de vie, mis en œuvre dans trente lycées en 2013. Il nous a paru en effet intéressant d'associer une démarche participative à une grande attente des jeunes, avec un budget maximum de 70000 euros par lycée. Cent trente lycées ont candidaté pour en bénéficier. Cette expérimentation va nous être utile pour penser les référentiels futurs des lycées. Je crois que ces lieux de socialisation et d'échanges sont extrêmement importants si l'on ne veut pas, comme l'a constaté Philippe Tournier, que les relations entre jeunes se limitent à des appels téléphoniques. Si on veut que le dialogue, la vie, l'échange se développent, il convient d'en créer les conditions et d'en faire un vrai sujet 
de travail et de réflexion. Du point de vue politique, si l'on tient à ce que le vivre ensemble se développe, cela ne peut se faire sans tenir compte des jeunes, sans être accompagnés par les équipes éducatives.

\section{QUELLES ÉVOLUTIONS DES ESPACES SCOLAIRES À L'AVENIR ?}

Luca Paltrinieri: La question des nouveaux apprentissages, notamment la question des TICE est soulevée depuis longtemps. Il est désormais courant d'évoquer l'émergence d'un nouveau modèle d'intelligence qu'on appelle l'intelligence connective. Les jeunes, les étudiants sont de plus en plus connectés entre eux et forment des communautés au sein de réseaux sociaux, qui sont eux-mêmes producteurs de savoirs. Jusqu'à présent, on a essayé de surveiller la diffusion de ces nouveaux savoirs, mais il semble inévitable qu'ils entraînent un changement dans la posture pédagogique des enseignants. Il s'agira sans doute davantage d'une posture d'accompagnement, l'enseignement servant à révéler un savoir qui est déjà là. Comment vont évoluer les espaces ? Pour forcer le trait, si l'on apprend en ligne, aura-t-on encore besoin d'école? Ma deuxième question porte sur le développement durable : en construisant des bâtiments à haute qualité environnementale, essaie-t-on d'éduquer les jeunes à un nouveau rapport à la nature et à l'écologie?

Philippe Tournier: L'une des motivations de la scolarisation a été, à l'origine, de libérer les autorités municipales de la question de savoir que faire des jeunes dans la journée. L'école n'avait pas seulement pour mission d'arracher la jeunesse à l'ignorance, mais aussi de la civiliser. Cette question est moins âpre aujourd'hui, quoi qu'on en dise. Chaque génération a toujours le sentiment de vivre une période extraordinaire, qui est une rupture définitive par rapport à celle qui précède. Mais on voit bien qu'effectivement, on ne maîtrise pas les contours d'un certain nombre d'évolutions, qu'on ne mesure pas leurs conséquences sur les rapports sociaux. On envisage souvent le futur comme une espèce de sur-présent parce qu'on duplique ce qu'on connaît. Au début du $\mathrm{XX}^{\mathrm{e}}$ siècle, on imaginait que des aéroplanes remplaceraient les vélos. Ce n'est pas ce qui s'est passé. L'idée que les nouvelles technologies vont faire disparaître le groupe d'apprentissage est sans doute une idée fausse. Je me souviens d'avoir vu un petit fascicule édité aux débuts de la télévision, au début des années soixante, qui s’intitulait "La fin des professeurs ? ». Or la télévision n’a pas supprimé le professeur. Il en va de même pour les nouvelles technologies. Un jeune qui apprend n'est pas un adulte en formation continue. L'apprentissage est une dynamique de groupe car il n'est pas naturel d'apprendre lorsqu'il fait beau dehors et qu'on a 14 ans. Au sein de ce groupe, que le professeur fasse un cours magistral ou qu'il travaille de manière autonome sur Internet, ce sont des formes 
de transmission du savoir liées à l'évolution des technologies mais la réalité fondamentale, c'est que l'apprentissage se fait mieux en groupe, on le sait depuis longtemps. Au XVII ${ }^{\mathrm{e}}$ siècle, pour la formation des futurs souverains, on allait chercher les enfants des domestiques pour former un groupe. Quand l'imprimerie est apparue, la classe n'a pas disparu. En revanche, la question du rythme, du temps est un problème. En France, l'objectif qu'on se fixe et qu'une partie des professeurs réussit à atteindre, est de canaliser l'attention de trente adolescents pendant 55 minutes. Ailleurs, on y a renoncé car personne ne peut être attentif à une tâche pendant 55 minutes d'affilée.

Florence Robine: Je suis convaincue qu'un certain nombre de formes scolaires traditionnelles sont encore pertinentes et que travailler au sein d'un groupe, avec un enseignant, sur des activités qui sont dirigées vers un objectif pédagogique clair, ont encore leur sens à l'école. Pour autant, d'autres formes d'apprentissage nécessitent d'autres formes d'espaces, physiques, virtuels, de coopération entre des individus à qui jusqu'à présent on ne laissait pas la possibilité de coopérer. Je vois par exemple émerger, dans un certain nombre de classes, des réseaux sociaux informels entre les élèves, auxquels participent aussi les enseignants, et qui constituent, à l'évidence, une véritable activité pédagogique, un véritable apprentissage coopératif entre des individus dont le positionnement diffère totalement de celui qu'on voit dans la salle de classe. L'enseignant n'est plus le leader, il n'est plus le seul vers lequel on regarde : il est l'un des membres de la communauté apprenante, avec des responsabilités et des compétences autres, et les élèves participent, finalement, presque à voix égale avec l'enseignant. Ces nouvelles formes d'espaces, aussi bien physiques que virtuelles, sont très différentes. On voit aussi émerger d'autres types de compétences. En France, le statut des professeurs documentalistes est en train d'évoluer considérablement. La mise au cœur d'un certain nombre d'établissements, y compris du point de vue architectural, du bâtiment de l'ancien CDI comme centre de connaissance et de culture, avec un professeur capable de mettre en relation les disciplines, les temps d'apprentissage, d'accompagner un travail de collaboration, une recherche documentaire, un travail virtuel, etc., a bouleversé un certain nombre de représentations et d'organisations pédagogiques.

Il est certain qu'on a toujours l'impression d'être plongé dans le maelström d'un changement de paradigmes mais il me semble que l'arrivée massive des nouvelles technologies de l'information, des réseaux sociaux, de cette diversité de coopérations et de sources possibles constitue véritablement un autre mode d'organisation, dont on va devoir tenir compte concrètement dans les établissements. C'est déjà le cas, par exemple, du rôle du wifi. Je constate que les seuls à ne pas être connectés, à l'heure actuelle, au monde extérieur dans les salles de classe, ce sont les professeurs. Tous les élèves ont dans leur trousse leur petit téléphone portable, ou leur mini tablette connectée en permanence sur 
le monde extérieur. Le professeur est devant des élèves dont les antennes sont dirigées vers leurs camarades, vers ceux qui sont à l'extérieur et vers les autres sources d'informations. Cela fait disparaître les murs de la salle de classe.

Luca Paltrinieri : Ce nouveau fonctionnement aura-t-il un impact sur l'architecture des classes, voire des écoles?

Bernard Quirot : Je crains de vous décevoir avec ma réponse : si nos débats montrent que les pratiques évoluent - de manière assez ténue, il ne s'agit sans doute pas d'une révolution - l'espace pédagogique n'évolue lui-même pratiquement pas, en tout cas guère plus que la constitution des villes depuis la naissance de l'urbanisme. Il y a une espèce de résistance des formes. D'ailleurs, l'architecture est-elle là pour d'évoluer en fonction des pratiques? Je n'en suis pas sûr. Ce que montre notre discussion, du point de vue de mon métier d'architecte, c'est que les choses évoluent très peu. S'il y a eu en effet une profonde transformation des espaces autrefois considérés comme annexes et qui sont aujourd'hui des espaces principaux, comme celui du CDI, on constate cependant une assez faible interaction entre l'espace et l'évolution des pédagogies ou des pratiques.

Il faut dire aussi que l'architecte intervient à la fin, et c'est peut-être là le problème. La rédaction du programme est une étape qui fige les choses au lieu de les ouvrir. La concertation intervient avant le projet d'architecture luimême : en définitive, le projet est là pour exécuter un programme qui a été prédéfini, même s'il reste évidemment un espace d'interprétation. À cela s'ajoute le fait que le maître d'ouvrage n'est pas toujours enclin à faire évoluer son programme par la suite, pour de nombreuses raisons, et notamment par crainte de recours en justice. La construction ou la restructuration d'un établissement engage une logistique lourde (concertation, programme, concours...) qui fait qu'il est très difficile, ensuite, d'apporter tout ce qu'un vrai projet d'architecture peut offrir.

Maurice Mazalto : L'architecture doit-elle précéder, suivre, accompagner les évolutions constatées?

Henriette Zoughebi : C'est un mouvement dialectique, fait de tensions et de frottements. Pour ma part, je connais des exemples de réelle interprétation de la part des architectes, et nous avons besoin de ce travail. Les jurys réunissent des proviseurs mais aussi des élèves et la collectivité, et je pense qu'il ne faut pas opposer d'un côté les programmes, et de l'autre les architectes.

On a parlé des perspectives sous l'angle éducatif et pédagogique, mais il y a aussi de nouvelles possibilités du point de vue des bâtiments et de l'architecture, de nouvelles formes sur lesquelles on peut travailler. Les nouveaux 
lycées doivent incarner l'inventivité, la créativité architecturale. L'architecture est une culture, ce n'est pas seulement une mise en œuvre. Je crois à l'architecture comme art, dans un contexte précis, de la même façon qu'il y a des arts appliqués, et je crois aussi que les jeunes des lycées en ont besoin. C'est un très grand enjeu : veut-on aller dans le sens de la marchandisation ou défendre une valeur de civilisation? L'éducation a un rôle fondamental à jouer de ce point de vue. Je considère que les évolutions actuelles ne sont pas des adaptations marginales mais une révolution, y compris du point de vue cognitif. Si la mission de l'école est bien l'entrée dans les savoirs et dans le partage des connaissances, il faut en faire une priorité. On n'a pas le choix. C'est une question de civilisation, de choix de société. Je crois que la possibilité du partage et de la coopération est à l'ordre du jour, que l'école continue à la maison, dans la rue. Il faut en tenir compte et prendre nos responsabilités: si nous voulons former des personnes libres, il faut que l'école, y compris dans ses formes matérielles, le prenne en compte. Concrètement, comment vont changer le couloir, la porte, le CDI ? Va-t-on créer la possibilité de s'installer sur des marches ? Comment prendre en compte les espaces libres, et pas seulement la cour de récréation ? C'est là-dessus qu'on peut travailler de manière positive.

Bernard Quirot : J'approuve ce qui vient d'être dit. Toutes ces évolutions vont dans le bon sens, mais elles ont une influence extrêmement ténue sur le bâtiment. Un bon bâtiment est un bâtiment qui a su évoluer, prendre plusieurs fonctionnalités au cours de sa vie. Si, justement, il a cette dignité qui a été évoquée tout à l'heure, s'il est bien construit, bien proportionné, avec des espaces dont la hauteur dépend de leur surface, s'il fait son travail de bâtiment, d'architecture, ces évolutions y trouveront leur place. Réfléchir au dessin de nouveaux gradins pour accueillir les enfants avec leurs portables ou leurs tablettes est secondaire. Si l'architecture est bien de l'architecture, elle s'y prêtera. Je pense que le lien entre l'évolution de l'espace et l'évolution des pratiques est très faible. Pour en donner un exemple, la vie urbaine a énormément changé, ne serait-ce qu'en matière de circulation, et pourtant la forme des villes a peu évolué. Il faut bien constater, la relative stabilité des espaces pédagogiques. Le métier d'architecte consiste à offrir le cadre le plus neutre, le plus beau, le mieux proportionné possible : un cadre à la fois pérenne et ouvert aux changements, qui ne sont eux-mêmes pas nécessairement traduits par la forme architecturale.

\section{REMARQUES FINALES}

Florence Robine : Ma vision est très liée à une préoccupation éducative autour des équipes elles-mêmes : comment faire en sorte que les équipes pédagogiques, la communauté éducative, comprenant évidemment les élèves et leurs familles, soient partie prenante de la réflexion sur les évolutions et les adaptations des espaces scolaires? Cet objectif me semble le plus important, car il tient 
non seulement à la qualité de vie à l'intérieur de l'école mais à des objectifs éducatifs de très haut niveau. Il s'agit de la responsabilité sociale et politique qu'a l'école dans l'émergence d'une nouvelle citoyenneté et, pour moi, c'est peut-être l'enjeu principal qui se dessine, à une époque où cette question devient extrêmement prégnante dans un monde globalisé, connecté, de plus en plus individualiste, dans lequel on a du mal à situer la véritable plus-value de la société. Je pense que c'est le cœur de la problématique de l'école elle-même, et donc des espaces scolaires.

Philippe Tournier: Il faudra des décennies pour que les 6,5 millions de mètres carrés de la Région Île-de-France soient transformés, et quand les transformations seront terminées, il y aura de nouvelles transformations en cours. Tel qu'il est, le bâti est une réalité et les bâtiments, même extraordinaires, que l'on construira, seront toujours marginaux. La scolarité se fera dans les bâtiments tels qu'ils sont aujourd'hui. Les bâtiments ne sont pas une entrave à l'évolution pédagogique. Si l'école n'évolue pas, c'est pour des raisons qui n'ont rien à voir avec le bâti dans lequel elle se trouve.

Bernard Quirot : Y-a-t-il d'autres expériences plus novatrices dans les autres pays? J'ai pu paraître pessimiste, au cours de cet échange, mais je regrette qu'il n'y ait pas de nouvelles expérimentations, même si l'on sait bien qu'elles seront de toute façon marginales. Nous aurions pu évoquer la période d'expérimentation sur l'espace et la pédagogie qui s'est mise en place à une certaine époque, et dont on ne parle plus aujourd'hui. On a l'impression d'être enfermés dans un programme-type et dans ce noyau dur de la salle de classe. Peut-être faudrait-il à nouveau tenter d'autres choses? Je crois en définitive que l'espace architectural ne peut pas suivre l'évolution des pratiques pédagogiques. Il peut les favoriser, en autorisant par exemple suffisamment de flexibilité, et plus sûrement encore en étant généreux et "digne », pour citer madame Zoughebi. La bonne architecture est celle qui se montre capable d'accueillir des évolutions parfois considérables, dans la durée de son existence.

Henriette Zoughebi : Si l'architecture est avant tout de l'espace et non de la décoration, si l'espace est bien pensé et qu'il est noble, au sens où on l'a défini tout à l'heure, c'est parce qu'il donne de la dignité, avec de bons matériaux, et il peut être le cadre de développements nouveaux. L'enjeu de ce siècle est la réussite de tous les enfants et pas seulement d'une élite. C'est un changement de paradigme colossal dans la vision de l'école. Permettre que chaque enfant se sente bien au lycée, alors que précédemment il y avait plus ou moins sa place, c'est un défi tout à fait neuf. Ce qui m'intéresse, c'est que l'école soit celle de l'égalité et qu'on change le regard sur les jeunes qui y travaillent et qui y vivent. 
